\title{
Cystic Mesenteric Inflammatory Pseudotumor Associated to Multidrug-Resistant Meningeal Tuberculosis and a Cerebrospinal Fluid Shunt: An Unusual Case
}

\author{
Martha Lilia Tena-Suck ${ }^{\mathrm{a}, \mathrm{e}}$, Jose Luis Soto-Hernandez ${ }^{\mathrm{b}}$, Juan Pablo Gonzalez-Mosqueda ${ }^{\mathrm{c}}$, \\ Citlaltepetl Salinas-Lara ${ }^{\mathrm{a}}$, Manuel Castillejos-Lopez ${ }^{\mathrm{d}}$
}

\begin{abstract}
Abdominal masses in childhood have a very large spectrum of benign and malignant causes. Inflammatory pseudotumor (IP) arising from intra-abdominal sites has only rarely been designated previously in children. We report the case of a 21-year-old HIV-negative man, in whom a cystic mesenteric IP appeared after 46 months of diagnosis of tuberculous meningitis (TBM) complicated with brain infarcts, tuberculoma, hydrocephalus and multiple neurosurgical procedures including ventriculoperitoneal (VP) cerebrospinal fluid (CSF) shunt placement, shunt exchanges and endoscopic ventricular septostomies. At diagnosis, primary antituberculous drugs were administered and mycobacterial CSF cultures were persistently negative; nevertheless, follow-up elevated adenosine deaminase levels in CSF suggested multidrug-resistant (MDR) tuberculosis and moxifloxacin and aminoglycoside were added with improvement. Repeated formation of abdominal cystic masses with shunt obstructions, abdominal distention and pain lead us to conclude that the peritoneum was no longer useful for CSF derivation and a ventriculo-atrial shunt was placed. After laparoscopic excision of an abdominal mass, microscopic examination showed a cystic tumor formed by proliferation of fibroblasts, myofibroblasts and inflammatory cells. Focally a granuloma with multinucleated giant cells (Langerhans cells) was observed and Ziehl-Neelsen staining showed acid-fast bacilli. Diagnosis of IP associated to MDR tuberculosis was made. This complication is probably under-recognized since histopathological study of abdominal masses related with CSF VP shunts is not performed systematically. Our case illustrates the importance of microscopic tissue examination to detect MDR and extensively drug-resistant (XDR) tuberculosis and the dif-
\end{abstract}

Manuscript accepted for publication May 18, 2015

aDepartment of Neuropathology, National Institute of Neurology and Neurosurgery, Mexico City, Mexico

bService of Infectology, National Institute of Neurology and Neurosurgery, Mexico City, Mexico

'Service of Neurosurgery, National Institute of Neurology and Neurosurgery, Mexico City, Mexico

dUnit of Epidemiologic Surveillance, Institute of Respiratory Disease, Mexico City, Mexico

${ }^{e}$ Corresponding Author: Martha Lilia Tena-Suck, Department of Neuropathology, National Institute of Neurology and Neurosurgery, Mexico City, Mexico. Email:mltenasuck@gmail.com

doi: http://dx.doi.org/10.14740/jnr333w ficult treatment of these cases.

Keywords: Meningeal tuberculosis; Inflammatory pseudotumor; Mesenteric tumor; Multidrug-resistant

\section{Introduction}

The latest 2014 World Health Organization (WHO) report of global tuberculosis (TB) estimates that 9 million people developed TB in 2013 and that TB caused 1.5 million people deaths [1]. The $2014 \mathrm{WHO}$ report also states that drug-resistant TB is worsening with an estimated 480,000 new cases of multidrugresistant (MDR) TB in 2013 [1]. CNS TB is the most lethal and incapacitating form of extra-pulmonary $\mathrm{TB}$ and occurs in approximately $1 \%$ of the patients with active TB $[2,3]$. In high resources countries meningeal TB is related with immunosuppression and AIDS. Only $30 \%$ of patients with brain TB have a positive chest radiograph [4-8]. Absence of features of TB on chest X-rays should therefore not rule out the possible existence of brain TB [4-9]. Tuberculous meningitis (TBM) and tuberculoma (TBC) are the two most important manifestations of CNS TB. Intracranial tuberculoma may be solitary or multiple [1-4]. Solitary tuberculoma may be indistinguishable from cranial abscess or primary brain tumor [8]. Intracranial TBC might be difficult to diagnose when the patient has no evidence or history of tuberculous infection [4]. Inflammatory pseudotumor (IP) was described as a wide range of neoplastic and reactive lesions such as inflammatory myofibroblastic tumor [4-8], and these tumors suggest a chronic inflammatory condition [9-11].

Abdominal pseudotumor is a rare and distinct clinical entity. Retroperitoneal mass is an extremely rare manifestation of extrathoracic TB. Abdominal TB collectively refers to gastrointestinal, splenic, pancreatic, hepatobiliary and abdominal lymph node involvement $[8,12]$. Diagnosis of abdominal pseudotumor is often delayed which can produce serious complications [8].

We report a young male with TBM and multiple cerebrospinal fluid (CSF) ventriculoperitoneal (VP) shunt complications, in whom abdominal pain, abdominal distention and vomiting prompted the excision of an abdominal mass related to CSF VP shunt. The specimen was referred to histopatho- 

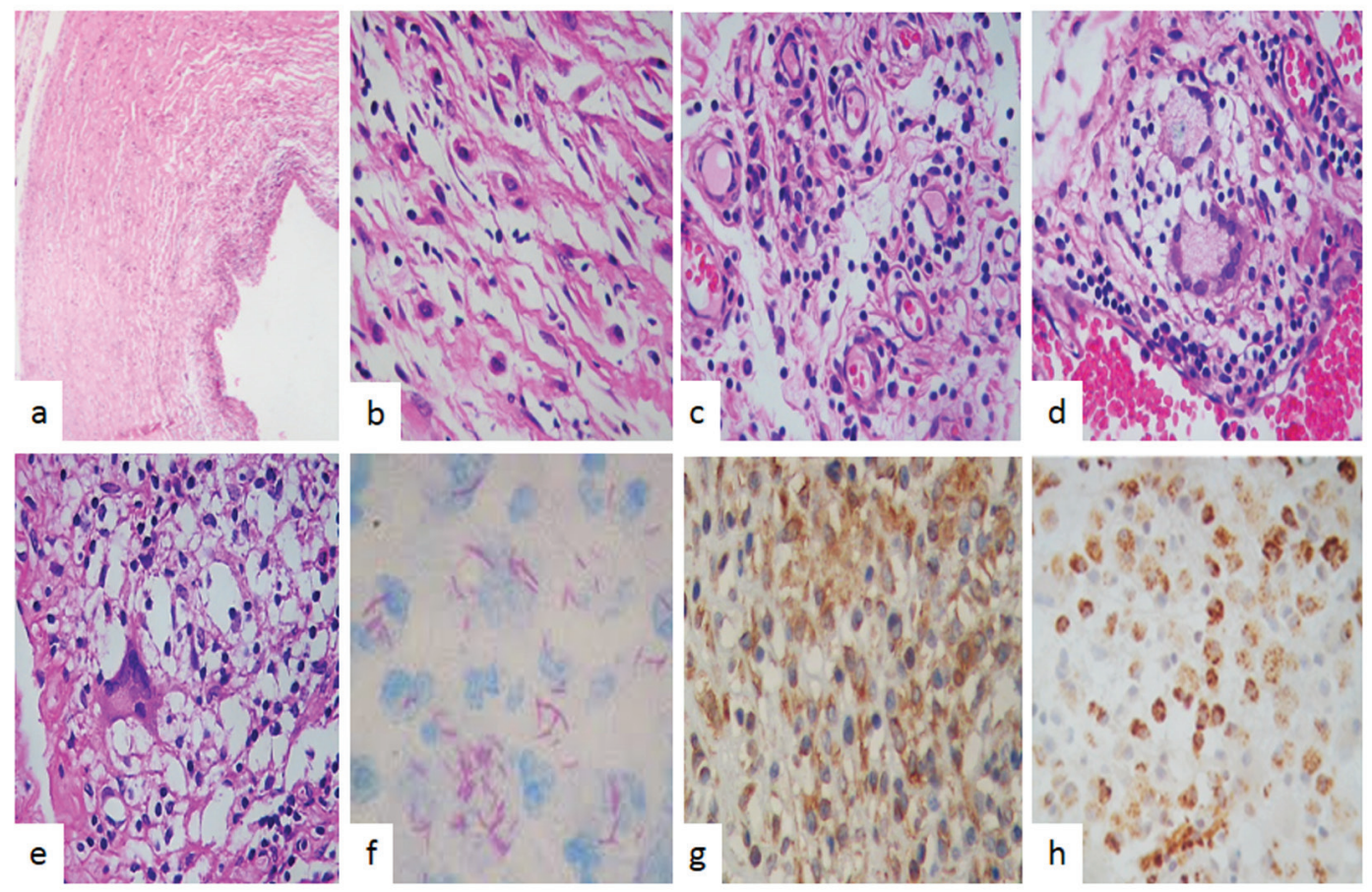

Figure 1. (a) Magnetic resonance imaging shows a mass of $15 \mathrm{~cm}$ in diameter. Axial T1W image shows well-circumscribed solid and heterogeneous mesenteric mass. (b) It might seem to have an eccentric scar, although calcification could also be possible. It is difficult to discern by magnetic resonance imaging. (c) Axial T2W fat sat image shows a large mesenteric mass. Notice the slightly decrease of signal intensity and the lack of a peripheral capsule. (d) Gross aspect of the tumor was cyst, the outer surface was soft whitish, and focally presents yellowish adipose tissue of normal characteristics. (e) Cut surface showed cellular debris. (f) The cyst wall was thin and homogeneous.

logical study with a diagnosis of malignancy, a mesenteric IP associated with TB which was found and pointed toward identifying MDR TB.

\section{Case Report}

A 17-year-old man presented with a 2-week history of headache, fever, nausea and unsteady gait. Upon admission neurological examination revealed confusion bilateral papilledema and meningeal signs. A chest X-ray was unremarkable and an HIV serum test was negative. Lumbar puncture showed an opening pressure of $250 \mathrm{~mm} \mathrm{H}_{2} \mathrm{O}$, and CSF analysis revealed a cell count of 187 cells $/ \mathrm{mm}^{3}$ ( $96 \%$ lymphocytes and $4 \%$ neutrophils), protein concentration of $676 \mathrm{mg} / \mathrm{dL}$ and $17 \mathrm{mg} / \mathrm{dL}$ of glucose with serum glucose of $90 \mathrm{mg} / \mathrm{dL}$. Adenosine deaminase CSF concentration was $22 \mathrm{UI} / \mathrm{L}$ (at our center $>7 \mathrm{UI} / \mathrm{L}$ is compatible with TBM); on microscopic examination CSF smear showed scant acid fast bacilli, bacterial, mycobacterial and fungal cultures remained negative. Antituberculous treatment was started with isoniazid, rifampin, pyrazinamide, intramuscular streptomycin $(30 \mathrm{~g})$ and dexamethasone. Admission brain CT scan showed brain edema. A cranial MRI performed 5 days after admission revealed contrast enhancement of basal cisterns and a small left basal ganglia infarction. His clinical condition improved and was discharged after 25 days in hospital. He continued supervised antituberculous drug treatment.
Six months later he presented with symptomatic intracranial hypertension and a CT scan showed hydrocephalus and a right VP shunt was placed. Eleven months after his first admission an MRI revealed new multiple bulbo pontine lesions with solid and ring enhancement after contrast administration compatible with brainstem tuberculoma. CSF remained inflammatory with ADA level of $37 \mathrm{UI} / \mathrm{L}$, and negative cultures. He was hospitalized and amikacin and moxifloxacin were added with clinical improvement. Thereafter multiple hospitalizations were required for a left CSF VP shunt placement and asymmetric hydrocephalus prompted an endoscopic ventricular anterior septostomy. At 27 months of follow-up an abdominal CT scan was performed by a new episode of hydrocephalus accompanied by abdominal distention revealing a giant abdominal pseudo cyst it was drained. Finally 46 months after first admission a new episode abdominal distention and pain was evaluated with a CT scan that revealed on right abdominal flank a $15 \mathrm{~cm}$ diameter mass with fluid density and some heterogeneous areas (Fig. 1c, d, f). We concluded that the peritoneum was no longer useful for CSF derivation and a ventriculo-atrial shunt was placed. Two weeks later the abdominal mass was excised by laparoscopy and macroscopic aspect suggested a cystic neoplasm. The tumor measure was $15 \times 10 \mathrm{~cm}$, external surface was smooth bright whitish, it has a homogeneous soft consistency and presented focal adipose tissue. The cut surface presented a yellowish fibrinoid necrotic material (Fig. 1d, e). The histopathological study revealed an inflamed tumor with 

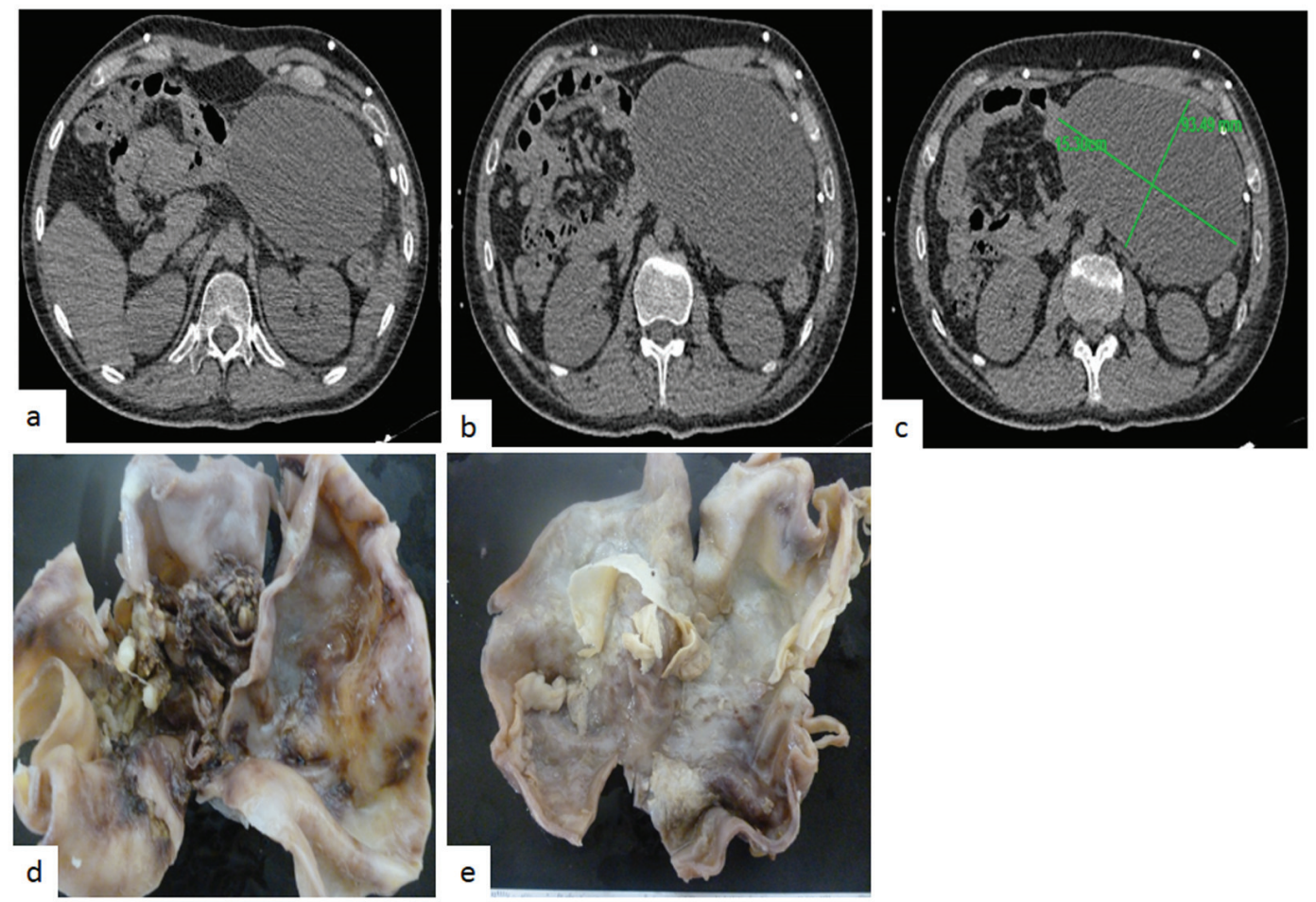

Figure 2. Pathologic findings. (a) Cyst tumor formed by spindle myofibroblastic cells in a fibromyxoid background with intermingled inflammatory cells $(H \& E, \times 200)$. (b) High power examination revealed a fibroblastic and myofibroblastic proliferation with lymphocytes and plasma cells and macrophages without caseating necrosis. (c) Vessels proliferations, lymphocytes and macrophages were observed (H\&E, $\times 400)$. (d) Giant cells and granuloma were also observed. (e) Giant cells and dirty macrophages that contained red bacillus, Zeel-Nielsen stain positive in (f). (g) Immunohistochemistry stain showed that spindle myofibroblastic cells were vimentin positive immunoreaction and in $(\mathrm{h})$ observed that the most common cells were macrophages which were strong positive to CD68 (IHQ, × 400).

a cystic IP (Fig. 2a), showing fibroblastic and myofibroblastic proliferation (Fig. 2b), inflammatory infiltrate of lymphocytes and plasma cells (Fig. 2c), and visible occasional eosinophils. In adipose tissue a granuloma was observed with giant cells of Langerhans type (Fig. 2d, e), and macrophages showed acid fast bacilli with Ziehl-Neelsen staining (Fig. 2f), without caseating necrosis. The tumor had a vimentin strongly positive immunoreaction (Fig. 2g) and desmin was negative. CD68 was strongly positive (Fig. 2h). The patient had an uneventful course and his case was presented to a committee for MDR and extensively drug-resistant (XDR) TB treatment.

\section{Discussion}

TBM is associated with significant neurological sequelae, high morbidity and a high mortality rate (WHO global tuberculosis report 2014) [1-4]. Present WHO recommendations make a distinction between new cases (not previously treated or for less than 30 days) and retreatment cases (longer than 30 days) [1]. New TB cases should be treated with a 6-month regimen of isoniazid, rifampicin, pyrazinamide, and ethambutol for the first 2 months (intensive phase), followed by isoniazid and rifampicin for the remaining 4 months (continuation phase) [11]. Extrapulmonary TB requires extension of continuation phase for a year [12]. As part of the new WHO end tuberculosis strategy (WHO global tuberculosis report 2014), universal drug-susceptibility testing (rapid or conventional) is strongly recommended before prescription of an antituberculosis regimen, although this is not universally available [11, 13]. In our patient multiple CSF cultures obtained at lumbar puncture or shunt placement and revision remained negative and susceptibility test was not possible [11, 13].

The importance of resistance of Mycobacterium tuberculosis isolates from the CSF to first-line antituberculosis drugs and lineage genotyping was demonstrated in a large series of patients enrolled in two studies of HIV-associated TBM in Ho Chi Minh City, Vietnam [14, 15]. Resistance test was available for 122 patients, the influence of antituberculosis drug resistance and M. tuberculosis lineage on 9-month mortality [15]. Isoniazid (INH) resistance without rifampin resistance was associated with increased mortality and multidrug resistance was uniformly fatal $[14,15]$.

Clinical presentation of IP depends on the site of the tumor. Mesenteric tumors usually present with a firm, smooth, painless mass associated with an inflammatory answer, which is categorized by fever, impaired growth, weight loss, anemia, elevated erythrocyte sedimentation rate, platelet count and hypergammaglobulinemia. Clinical status is the differential diagnosis of mesenteric IP from malignant mass lesions [12, 
16]. The abdomen is considered to be one of the most prevalent sites for manifestation of TB $[6,12]$, any abdominal organ may be involved in localization of $\mathrm{TB}$, but symptoms of abdominal pseudotumor are not specific and diagnosis can often be missed and mimic malignancy [7, 9-11, 13, 15]. Because of their polymorphic presentation physicians should be aware of this distinct entity and apply all diagnostic procedures. The clinical presentation is determined by the site of origin and the effects of the mass $[7,9-11,13,15]$ that arise in a variety of locations such as brain, bone, larynx, stomach, large intestine, small intestine, lung, liver, mediastinum, retroperitoneal and omentum which are mainly diagnosed in the first two decades of life and arise from multiple locations [5-7, 9, 12]. These tumors suggest a chronic inflammatory condition and presents with non-specific clinical symptoms and its diagnosis can be difficult $[5-7,9,12]$.

The WHO classification places inflammatory myofibroblastic tumors in an intermediate category because of tendency for a small risk of metastases and local recurrence [10, 11, 13]. These findings have recently shown that chromosomal abnormalities may be suggestive of clonal origin, not merely a reactive process and should be considered as a true neoplasm [10, 13].

Abdominal mass and retroperitoneal TB which have increased in recent years only contribute to $1 \%$ of clinical manifestations of disease [13, 16-20].

IP is composed of a proliferation of spindle-shaped cells arranged in the hyaline material with chronic inflammatory cells, composed mainly of plasma cells and lymphocytes [5, $6,12]$. Immunohistochemical tumor cells usually are positive for smooth muscle actin, and vimentin, and negative for desmin, CD117 (c-kit), anaplastic lymphoma kinase-1 [5, 6, 12]. Expression of anaplastic lymphoma kinase (ALK1) protein, immunohistochemistry can be helpful in making a correct diagnosis $[5,6,12]$.

Several benign fibroblastic lesions such as nodular fasciitis, leiomyoma, desmoids tumor and myofibroblastosis have been considered as differential diagnosis [7, 9-11, 13, 15].

Abdominal TB is rare even in endemic areas and is often mistaken for a malignant neoplasm $[5,6]$. Intra-abdominal IMTs have clinical importance due to their relationship to malignancy $[5,6]$. Intra-abdominal IMTs are difficult to distinguish preoperatively from other malignancies, such as sarcomas, lymphomas, and metastases $[5,6]$.

The differential diagnosis of mesenteric and retroperitoneal mass also included lymphoma, leiomyosarcoma, GIST, desmoid tumor, Wegener's granulomatosis, IP, and TB [13, 16, 17, 19-21].

This case presented meningeal tuberculosis and abdominal IP-associated TB, this combination is rare but some cases have been published [13, 16, 17, 19-21]. Vernaz et al [20] noted a case with frontal lesions suggestive of a tumor process and abdominal mass, with thoracoabdominal and mesenteric adenopathies. Hablani et al [17] reported the imaging features of four cases of abdominal pseudotumor TB in order to demonstrate diagnostic difficulties. The presentation of abdominal TB can be varied according to lesion size and localization [6, 12].

The etiology and pathogenesis of IP are not yet under- stood. Infection is one of the hypothesis because of the presence of granulomas and giant cells in the masses, as occurred in patients who have TB [7, 9-11, 13, 15]. Mycobacteria have been found in spindle cell pseudotumor (MSP). In up to $40 \%$ of cases, granulomata, giant cells and EBV are detected in the involved tissue [11]. IMT occurs after trauma, surgery or infection such as Epstein-Barr virus and human herpes virus related with reactive cytokine production $[10,11]$. In addition, it has been hypothesized that IP may have an autoimmune nature. Autoimmunity is suggested by the high plasmatic cell content in histologic specimens. Infection is one of the major theorizes because of the presence of granulomas and giant cells in the masses, as occurred in our patient who had TB [7, 9-11, 13, $15]$.

Clinicians should be aware of this unusual manifestation of TB which affects immunosuppressed patients but interestingly our patient had intact immune system. Processes such as granuloma formation and granuloma-induced angiogenesis provide niches and/or conditions in which mycobacteria can replicate and disseminate, eventually leading to successful host-to-host transmission vs. drug resistance [14, 15]. Identifying specific host processes on which pathogenic mycobacteria depend enables the rational development of host-directed therapies for TB $[14,15]$.

Cosma et al [22] suggested that homing of infected macrophages to sites of infection is a general feature of the pathogenesis of TB and has important consequences for therapeutic strategies.

We presented a rare case of meningeal tuberculosis treated and remained asymptomatic during 2 years, and suddenly he presented abdominal pain and a mesenteric mass that corresponded to an IP associated to TB. These findings have suggested new and specific host-directed therapies that await further clinical exploration.

\section{Conclusions}

Cystic mesenteric IP associated to MDR meningeal tuberculosis and a CSF shunt, is an unusual case. Our case illustrates the importance of microscopic tissue examination to detect MDR and XDR TB and the difficult treatment of these cases.

\section{Conflicts of Interest}

There is no conflict of interest.

\section{Grant Support}

None.

\section{Abbreviations}

WHO: World Health Organization; TB: tuberculosis; TBM: tuberculous meningitis; MDR: multidrug-resistant; IP: inflam- 
matory pseudotumor; CSF: cerebrospinal fluid; VP: ventriculo-peritoneal; MRI: magnetic resonance image; HIV: human immunodeficiency virus; MSP: spindle cell pseudotumor; EBV: Epstein-Barr virus; GIST: gastrointestinal stromal tumor

\section{References}

1. WHO, Global tuberculosis report 2014World Health Organization, Geneva (2014) http://www.who.int/tb/publications/global_report/en/ (accessed March 3, 2015).

2. Christensen A $\bar{S}$, Roed C, Omland LH, Andersen PH, Obel $\mathrm{N}$, Andersen AB. Long-term mortality in patients with tuberculous meningitis: a Danish nationwide cohort study. PLoS One. 2011;6(11):e27900.

3. Thwaites G, Fisher M, Hemingway C, Scott G, Solomon $\mathrm{T}$, Innes J. British Infection Society guidelines for the diagnosis and treatment of tuberculosis of the central nervous system in adults and children. J Infect. 2009;59(3):167187.

4. Blumberg HM, Burman WJ, Chaisson RE, Daley CL, Etkind SC, Friedman LN, Fujiwara P, et al. American Thoracic Society/Centers for Disease Control and Prevention/ Infectious Diseases Society of America: treatment of tuberculosis. Am J Respir Crit Care Med. 2003;167(4):603662.

5. Chanet V, Baud O, Deffond D, Romaszko JP, Beytout J. Pseudotumor presentation of intracerebral tuberculomas. South Med J. 2005;98(4):489-491.

6. Velasquez MJ, Szigethi QM, Panace VR, Morales IR, Marquez CS, Pefaur PJ, Mocarquer MA, et al. [Hepaticsplenic micobacteriosis, unusual form of probable extrapulmonary tuberculosis. Case report and review]. Rev Chilena Infectol. 2007;24(1):59-62.

7. Gleason BC, Hornick JL. Inflammatory myofibroblastic tumours: where are we now? J Clin Pathol. 2008;61(4):428-437.

8. Iype T, Chacko S, Raghavan S, Mathew R, Mohan M. Preliminary report of directly observed treatment, short course in tuberculous meningitis. Ann Indian Acad Neurol. 2010;13(1):57-60.

9. Ribeiro MC, Lopes LR, de Souza Neto JC, Meirelles LR, de Carvalho RB, Andreollo NA. Rare gastric inflammatory myofibroblastic tumor in an adult woman: a case report with review of the literature. Case Rep Med. 2012;2012:374070.

10. Coffin CM, Fletcher JA. Inflammatory myofibroblastic tumour. In: Fletcher CD, Unni KK, Mertens F, editors. World Health Organization classification of tumours Pathology and genetics of tumours of soft tissue and bone.
Lyon: IARC Press; 2002. pp. 91-93.

11. Oz Puyan F, Bilgi S, Unlu E, Yalcin O, Altaner S, Demir M, Cakir B. Inflammatory pseudotumor of the spleen with EBV positivity: report of a case. Eur J Haematol. 2004;72(4):285-291.

12. Golden MP, Vikram HR. Extrapulmonary tuberculosis: an overview. Am Fam Physician. 2005;72(9):1761-1768.

13. Kovach SJ, Fischer AC, Katzman PJ, Salloum RM, Ettinghausen SE, Madeb R, Koniaris LG. Inflammatory myofibroblastic tumors. J Surg Oncol. 2006;94(5):385-391.

14. Tho DQ, Torok ME, Yen NT, Bang ND, Lan NT, Kiet VS, van Vinh Chau N, et al. Influence of antituberculosis drug resistance and Mycobacterium tuberculosis lineage on outcome in HIV-associated tuberculous meningitis. Antimicrob Agents Chemother. 2012;56(6):3074-3079.

15. Zumla A, Chakaya J, Centis R, D'Ambrosio L, Mwaba P, Bates M, Kapata N, et al. Tuberculosis treatment and management--an update on treatment regimens, trials, new drugs, and adjunct therapies. Lancet Respir Med. 2015;3(3):220-234

16. Izzedine H, Servais A, Launay-Vacher V, Deray G. Retroperitoneal fibrosis due to Wegener's granulomatosis: a misdiagnosis as tuberculosis. Am J Med. 2002;113(2):164166.

17. Hablani N, Souei Mhiri M, Tlili Graies K, Jemni Gharbi H, Abdallah S, Bel Hadj Hamida R. [Pseudotumoral form of abdominal tuberculosis: report of four cases]. J Radiol. 2005;86(9 Pt 1):1021-1025.

18. Coffin CM, Hornick JL, Fletcher CD. Inflammatory myofibroblastic tumor: comparison of clinicopathologic, histologic, and immunohistochemical features including ALK expression in atypical and aggressive cases. Am J Surg Pathol. 2007;31(4):509-520.

19. Kim SJ, Kim WS, Cheon JE, Shin SM, Youn BJ, Kim IO, Yeon KM. Inflammatory myofibroblastic tumors of the abdomen as mimickers of malignancy: imaging features in nine children. AJR Am J Roentgenol. 2009;193(5):14191424.

20. Vernaz A, Enaud L, Blanc S, Stoven C, Tasset C, Losi $\mathrm{S}$, Andriolo E, et al. [Brain tuberculoma in a 10-yearold child: the diagnosis is in the belly]. Arch Pediatr. 2012;19(8):832-836.

21. Romand F, Gaudin JL, Bobichon R, Souquet JC. [Abdominal tuberculosis of pseudotumor aspect]. Presse Med. 1997;26(36):1717-1721.

22. Cosma CL, Humbert O, Sherman DR, Ramakrishnan L. Trafficking of superinfecting Mycobacterium organisms into established granulomas occurs in mammals and is independent of the Erp and ESX-1 mycobacterial virulence loci. J Infect Dis. 2008;198(12):1851-1855. 